\title{
Sala de aula invertida e aprendizagem baseada em problemas em design: uma revisão sistemática da literatura
}

\author{
Flipped classroom and problem-based learning in design: a systematic \\ literature review
}

\author{
Ana Carolina dos Santos Machado, Elton Rubens Vieira da Silva e \\ Felipe Pessoa Tejo Gabriele
}

sala de aula invertida, aprendizagem baseada em problemas, ensino, revisão sistemática da literatura

\begin{abstract}
Este artigo apresenta os resultados da Revisão Sistemática da Literatura, realizada entre 2019 e 2020 no Projeto de Pesquisa intitulado "Sala de Aula Invertida e Aprendizagem Baseada em Problemas no ensino de Computação Gráfica e Design", com o objetivo de analisar o uso de tais metodologias ativas em cursos de design e áreas afins e verificar se há aumento de desempenho dos estudantes na assimilação do conteúdo e em atividades acadêmicas e/ou profissionais. Realizou-se buscas em 5 bases de dados diferentes com descritores e filtros específicos, além de critérios para inclusão e exclusão de artigos e os resultados obtidos através da catalogação das experiências pedagógicas foram estudados a partir do método da Análise de Conteúdo. Dos principais resultados, destacam-se o pouco estudo na área de atuação e a insegurança do estudante com métodos diferentes do tradicional, criando algumas barreiras e quedas de desempenho durante as primeiras fases da implementação, contornadas posteriormente, permitindo um aumento gradativo do desempenho.
\end{abstract}

flipped classroom, problem-based learning, teaching, systematic literature review

This article presents the results of the Systematic Literature Review, carried out between 2019 and 2020 in the Research Project entitled "Flipped Classroom and Problem-Based Learning in the teaching of Computer Graphics and Design", in order to analyze the use of such active methodologies in design courses and related areas and to check if there is an increase in student performance in the assimilation of content and in academic and / or professional activities. Searches were carried out in 5 different databases with specific descriptors and filters, in addition to criteria for inclusion and exclusion of articles and the results obtained through the cataloging of pedagogical experiences were studied using the Content Analysis method. Of the main results, we highlight the little study in the area of activity and the insecurity of the student with methods other than the traditional one, creating some barriers and performance drops during the first phases of implementation, but which are circumvented, allowing for a gradual increase later.

\section{Introdução}

Em face ao avanço da tecnologia que vivenciamos, a área da educação está passando por uma revolução em seu conteúdo e forma. Em relação ao conteúdo, a quantidade de informações que temos de fácil acesso aumenta rápida e exponencialmente. O conhecimento,

Anais do $10^{\circ} \mathrm{CIDI}$ e $10^{\circ} \mathrm{CONGIC}$

Kelli C.A.S. Smythe, Rafael de Castro Andrade (orgs.)

Sociedade Brasileira de Design da Informação - SBDI

Curitiba | Brasil | 2021
Proceedings of the $10^{\text {th }} \mathrm{CIDI}$ and $10^{\text {th }}$ CONGIC

Kelli C.A.S. Smythe, Rafael de Castro Andrade (orgs.)

Sociedade Brasileira de Design da Informação - SBDI Curitiba | Brazil | 2021 
que antes era relegado aos professores e mestres, hoje pode ser acessado por qualquer pessoa utilizando um link de internet. A escola deixou de ser o foco de transmissão de conhecimento, perdendo assim sua posição de curador das informações que seriam transmitidas para as próximas gerações.

Já a forma de como se faz a educação também mudou. As metodologias tradicionais de ensino já na década de 70 eram reconhecidas como passivas e pouco estimulantes (Freire, 2003). Novas metodologias estão sendo utilizadas, com avanços significativos na utilização das novas ferramentas tecnológicas e da internet. Streaming de vídeos, podcasts, games, plataformas de comunicação síncrona, fóruns de discussão, redes sociais dos mais diversos fins, todas essas ferramentas podem e devem ser utilizadas com o objetivo de aproximar o conhecimento aos estudantes. $O$ desafio é promover reformas na educação que acompanhem o desenvolvimento tecnológico, científico, social, econômico, cultural e ambiental (Souza \& Dourado, 2015).

Sala de Aula Invertida e Aprendizagem Baseada em Problemas são duas metodologias que subvertem a forma tradicional de ensino e fazem uso das novas tecnologias para tornar a aprendizagem mais dinâmica (Jensen, 2017). A metodologia Sala de Aula Invertida permite uma combinação das teorias behaviorista e construtivista de ensino (Bishop, 2003), pois preconiza que o conteúdo da aula deve ser transmitido para os estudantes antes da aula presencial, fazendo uso de todas as ferramentas tecnológicas comunicacionais que os professores tiverem acesso. Em paralelo, a Aprendizagem Baseada em Problemas é uma abordagem pedagógica que permite ao estudante aprender enquanto está engajado na resolução de problemas. Aos estudantes é dada a oportunidade de resolver problemas de forma colaborativa, criando modelos mentais para aprendizagem, de forma autodirigida, criando hábito de prática e reflexão.

O ensino de design possui uma complexidade inerente à área, devido ao seu caráter dinâmico de atuação. Para Ezio Manzini (2017), a época de transição que vivemos exige do designer uma compreensão profunda das dinâmicas sociotécnicas. Essa compreensão do design evidencia uma necessidade de reposicionamento da disciplina em um contexto de complexidade causada pelas mudanças sociais e tecnológicas.

A fim de compreender a utilização das metodologias SAl e ABP nos cursos de Design, realizamos uma revisão sistemática da literatura com o objetivo de analisar trabalhos publicados sobre a aplicação dos novos métodos de ensino pelos professores e estudantes de Design e analisar os resultados desses estudos.

\section{Metodologia}

Uma revisão sistemática da literatura, assim como outros tipos de revisão, é um tipo de pesquisa que utiliza como fonte de dados estudos sobre determinada área do conhecimento. Esse tipo de pesquisa apresenta um resumo de evidências coletadas através de uma estratégia definida de maneira explícita e sistematizada de busca, apreciação crítica e síntese 
da informação (Sampaio \& Mancini, 2007). Como apresentado na figura 1, Sampaio e Mancini (2007) propõe os seguintes passos metodológicos para a revisão sistemática da literatura: definição da pergunta, busca das evidências, revisão e seleção dos estudos, analise da qualidade dos estudos e aprendizagem com os resultados. As revisões da literatura são considerados estudos secundários, que têm nos estudos primários sua fonte de dados. Assim, entende-se como fontes primárias os artigos científicos publicados que relatam os resultados de pesquisas realizadas sobre o tema principal da revisão (Galvão \& Pereira, 2014).

Figura 1: descrição geral sobre o processo de Revisão Sistemática da Literatura.

Fonte: Sampaio \& Mancini (2007).

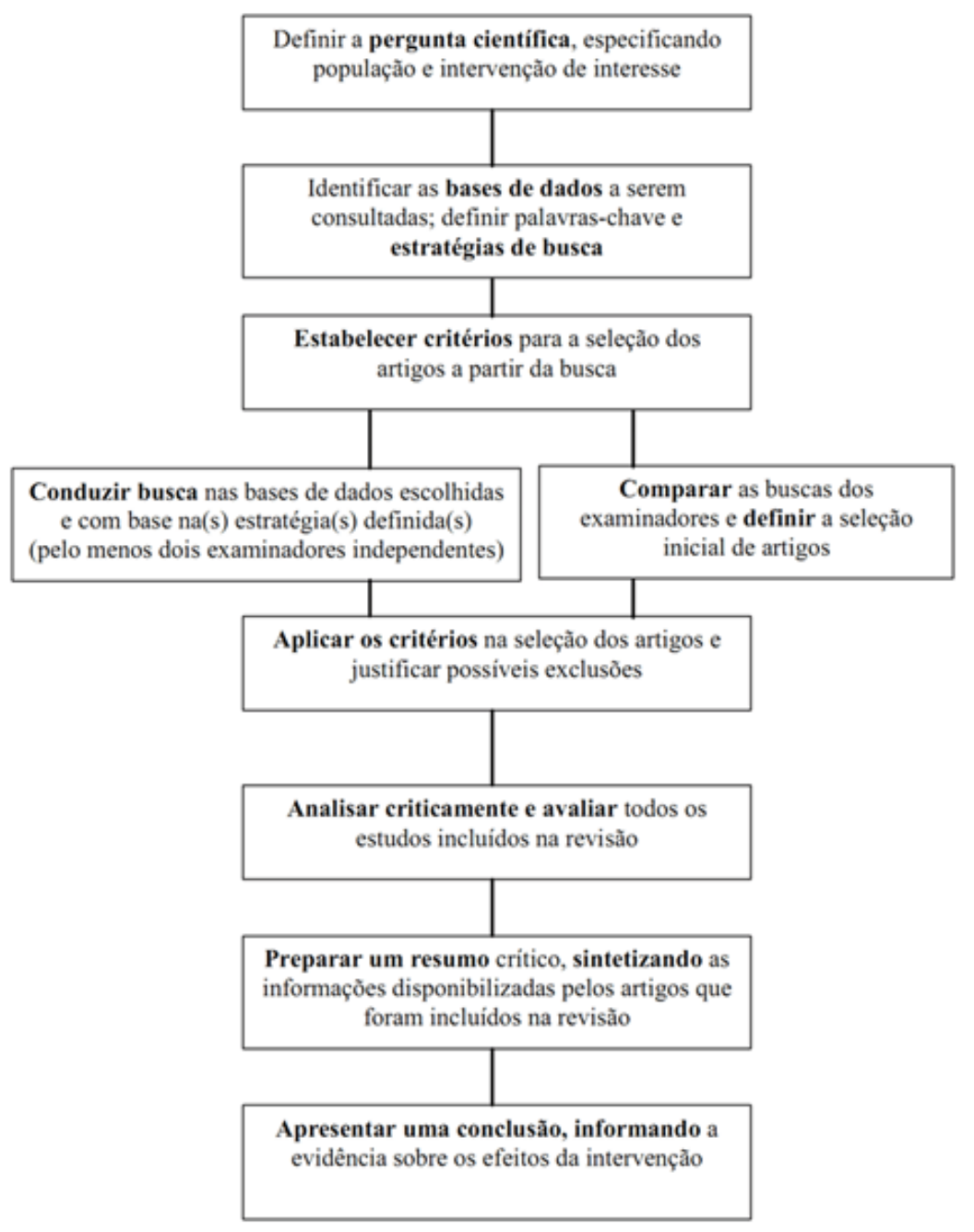

Antes de iniciar uma revisão sistemática da literatura, três etapas precisam ser consideradas: definir o objetivo da revisão, identificar a literatura e selecionar os estudos possíveis de serem incluídos. A partir destes pressupostos, o objetivo da revisão foi identificar a utilização dos métodos de Sala de Aula Invertida e Aprendizagem Baseada em Problemas em cursos técnicos e superiores em Design. As bases de dados utilizadas na execução da busca foram o SciElo, o Periódicos CAPES, o Google Scholar, o SpringerLink e o ERIC. 
A Revisão Sistemática da Literatura foi aplicada com a participação de dois pesquisadores bolsistas de iniciação científica, que avaliaram de forma independente cada artigo, a partir de um protocolo estabelecido de: como os artigos são encontrados; critérios de inclusão/exclusão; definição de interesse dos resultados; clareza dos resultados; qualidade do estudo; e análise estatística.

Em cada base de dados foi realizada busca com as palavras-chave "Sala de Aula Invertida" e "Aprendizagem Baseada em Problemas". Tais palavras-chaves foram escolhidas por serem o nome pelo qual é chamada as metodologias de ensino, nenhuma outra palavra chave foi escolhida como busca primária, pois a intenção do primeiro filtro foi a ampliação do alcance dos resultados. A busca se deu com as palavras chaves associadas e cada termo individualmente, podendo aparecer no título, resumo ou assunto. As palavras chaves também seriam traduzidas para sua versão em inglês para ampliar o alcance da busca. Definiu-se ainda que seriam critérios de inclusão da pesquisa secundária artigos relacionados à implementação dos métodos da SAl e da ABP e seus resultados em cursos de design ou áreas afins, ou implementação em cursos técnicos ou equivalentes. Os critérios de inclusão dos resultados foram selecionados para focalizar os estudos na área de Design e também foi condicionado a seleção para textos que estão disponíveis completos e abertos para a comunidade acadêmica. Foram considerados critérios de exclusão: artigos com mais de 5 anos, pois com tema do estudo apresenta alto potencial de evolução, graças aos avanços tecnológicos na área de comunicação; textos incompletos ou repetidos, quando o mesmo estudo ou partes do mesmo estudo são publicados em veículos de divulgação diferentes e; aqueles que não traziam a discussão da implementação dos métodos em um curso, onde a discussão no texto era apenas teórica.

$\mathrm{Na}$ segunda etapa da pesquisa realizou-se a separação dos artigos em duas pastas: artigos que mencionam a implementação dos métodos SAI e ABP em cursos de design ou áreas afins e artigos que mencionam a implementação dos métodos em cursos técnicos ou médios. A identificação dos artigos selecionados se deu através da leitura dos resumos dos textos selecionados na primeira etapa do processo de revisão sistemática da literatura.

$\mathrm{Na}$ última fase analisou-se os resultados levantados a partir da utilização das metodologias SAl e ABP. Os resultados obtidos através da catalogação das experiências pedagógicas foram estudados utilizando os conceitos da Análise do Conteúdo. Nesta fase, identificamos ainda a classe do artigo, título, objetivo, método de pesquisa utilizado, resultados alcançados e análise resumida.

\section{Resultados e Discussões}

Iniciou-se a revisão com a busca primária. A primeira pesquisa foi na plataforma da SciElo, a qual fornece as opções de consulta: palavra-chave de busca, índice de busca, filtros e resultados. Foram encontrados 50 artigos por meio da busca básica com as palavras "sala de aula invertida" ou "aprendizagem baseada em problemas"; índice de busca por Resumo; Idioma (português ou inglês), até 5 anos, artigos. Ao substituir o índice de "Resumo" para 
"Título", foram obtidos apenas 14 artigos. Já na busca com as palavras "flipped classroom" ou "problem-based learning"; índice de busca por Resumo; Idioma (português ou inglês), até 5 anos, artigos; foram encontrados 60 artigos. Substituindo nesta última pesquisa o índice para "Título", surgiram 29 artigos.

Tabela 1: Pesquisa primária - resultados da pesquisa no SciElo. Fonte: elaboração própria.

\begin{tabular}{|c|c|c|c|c|c|}
\hline $\begin{array}{c}\text { PALAVRA-CHAVE DE } \\
\text { BUSCA }\end{array}$ & $\begin{array}{l}\text { ÍNDICE } \\
\text { DE } \\
\text { BUSCA }\end{array}$ & FILTROS & RESULTADOS & LINK & OBSERVAÇÕES \\
\hline $\begin{array}{l}\text { "sala de aula invertida" } \\
\text { OU "aprendizagem } \\
\text { baseada em problemas" }\end{array}$ & Resumo & $\begin{array}{l}\text { Idioma } \\
\text { (português } \\
\text { ou inglês); } \\
\text { Até } 5 \text { anos; } \\
\text { Artigos }\end{array}$ & 50 & $\begin{array}{c}\text { BUSCA } \\
1\end{array}$ & $\begin{array}{l}\text { Na planilha indica } \\
50 \text { resultados. } \\
\text { Pesquisei } \\
\text { diretamente na } \\
\text { pasta da BUSCA 1, } \\
\text { pois não estava } \\
\text { tendo acesso ao } \\
\text { ícone. A pasta tinha } \\
\text { apenas } 49 \text { artigos } \\
\text { para analisar. }\end{array}$ \\
\hline $\begin{array}{l}\text { "sala de aula invertida" } \\
\text { OU "aprendizagem } \\
\text { baseada em problemas" }\end{array}$ & Título & $\begin{array}{l}\text { Idioma } \\
\text { (português } \\
\text { ou inglês); } \\
\text { Até } 5 \text { anos; } \\
\text { Artigos }\end{array}$ & 14 & $\begin{array}{c}\text { BUSCA } \\
2\end{array}$ & $\begin{array}{l}\text { Na planilha indica } \\
13 \text { resultados, } \\
\text { porém quando eu } \\
\text { clico no ícone } \\
\text { BUSCA } 2 \text {, } \\
\text { aparecem } 14 \\
\text { resultados no site do } \\
\text { Scielo. }\end{array}$ \\
\hline $\begin{array}{l}\text { "flipped classroom" OU } \\
\text { "problem-based learning" }\end{array}$ & Resumo & $\begin{array}{l}\text { Idioma } \\
\text { (português } \\
\text { ou inglês); } \\
\text { Até } 5 \text { anos; } \\
\text { Artigos }\end{array}$ & 60 & $\begin{array}{c}\text { BUSCA } \\
3\end{array}$ & $\begin{array}{l}\text { Na planilha indica } \\
55 \text { resultados, } \\
\text { porém quando eu } \\
\text { clico no ícone } \\
\text { BUSCA 3, } \\
\text { aparecem } 60 \\
\text { resultados no site do } \\
\text { Scielo. }\end{array}$ \\
\hline $\begin{array}{l}\text { "flipped classroom" OU } \\
\text { "problem-based learning" }\end{array}$ & Título & $\begin{array}{l}\text { Idioma } \\
\text { (português } \\
\text { ou inglês); } \\
\text { Até } 5 \text { anos; } \\
\text { Artigos }\end{array}$ & 29 & $\begin{array}{c}\text { BUSCA } \\
4\end{array}$ & $\begin{array}{l}\text { Na planilha indica } \\
28 \text { resultados, } \\
\text { porém quando eu } \\
\text { clico no ícone } \\
\text { BUSCA 4, } \\
\text { aparecem } 29 \\
\text { resultados no site do } \\
\text { Scielo. }\end{array}$ \\
\hline
\end{tabular}

TOTAL
SCIELO:

Na segunda plataforma, CAPES, com a busca das palavras "sala de aula invertida" ou "aprendizagem baseada em problemas"; no índice Título; Idioma (português ou inglês), até 5 anos, foram encontrados 23 artigos. Na busca com as palavras "flipped classroom" ou "problem-based learning"; no índice Título; Idioma (português ou inglês), até 5 anos, foram encontrados 203 artigos. Nesta plataforma não havia opção de índice de busca por Resumo. Salientamos ainda que, nas buscas onde foram encontrados os artigos, realizou-se ainda um 
refino revisado por pares; tópicos problem-based learning e teaching methods como critérios de inclusão, e artigos de outras áreas como critério de exclusão já utilizados na busca primária, devido ao alto resultado de artigos (1.647).

Tabela 2: Pesquisa primária - resultados da pesquisa na Capes. Fonte: elaboração própria.

\begin{tabular}{|c|c|c|c|c|c|}
\hline $\begin{array}{l}\text { PALAVRA-CHAVE } \\
\text { DE BUSCA }\end{array}$ & $\begin{array}{l}\text { ÍNDICE } \\
\text { DE } \\
\text { BUSCA }\end{array}$ & FILTROS & $\begin{array}{l}\text { RESUL- } \\
\text { TADOS }\end{array}$ & LINK & OBSERVAÇÕES \\
\hline $\begin{array}{l}\text { "sala de aula invertida" } \\
\text { OU "aprendizagem } \\
\text { baseada em problemas" }\end{array}$ & Resumo & $\begin{array}{l}\text { Idioma (português ou } \\
\text { inglês); Até } 5 \text { anos; } \\
\text { Artigos }\end{array}$ & & $\begin{array}{c}\text { BUSCA } \\
5\end{array}$ & $\begin{array}{l}\text { Não existe esta opção de } \\
\text { índice de busca }\end{array}$ \\
\hline $\begin{array}{l}\text { "sala de aula invertida" } \\
\text { OU "aprendizagem } \\
\text { baseada em problemas" }\end{array}$ & Título & $\begin{array}{l}\text { Idioma (português ou } \\
\text { inglês); Até } 5 \text { anos; } \\
\text { Artigos }\end{array}$ & 23 & $\begin{array}{c}\text { BUSCA } \\
6\end{array}$ & $\begin{array}{l}\text { Refino de busca: revisado } \\
\text { por pares; idioma português }\end{array}$ \\
\hline $\begin{array}{l}\text { "flipped classroom" or } \\
\text { "problem-based } \\
\text { learning" }\end{array}$ & Resumo & $\begin{array}{l}\text { Idioma (português ou } \\
\text { inglês); Até } 5 \text { anos; } \\
\text { Artigos }\end{array}$ & & $\begin{array}{c}\text { BUSCA } \\
7\end{array}$ & $\begin{array}{l}\text { Não existe esta opção de } \\
\text { índice de busca }\end{array}$ \\
\hline
\end{tabular}

\begin{tabular}{|c|c|c|c|c|c|}
\hline $\begin{array}{l}\text { "flipped classroom" or } \\
\text { "problem-based } \\
\text { learning" }\end{array}$ & Título & $\begin{array}{l}\text { Idioma (português ou } \\
\text { inglês); Até } 5 \text { anos; } \\
\text { Artigos }\end{array}$ & 203 & $\begin{array}{c}\text { BUSCA } \\
8\end{array}$ & $\begin{array}{l}\text { Refino de busca: revisado } \\
\text { por pares; tópicos problem- } \\
\text { based learning e teaching } \\
\text { methods como critérios de } \\
\text { inclusão e artigos de outras } \\
\text { áreas como critério de } \\
\text { exclusão já utilizados na } \\
\text { busca primária pelo alto } \\
\text { resultado de artigos (1.647) }\end{array}$ \\
\hline
\end{tabular}

TOTAL CAPES:

226

Já na terceira e na quinta plataforma, o Google acadêmico e o SpringerLink, os resultados não foram considerados, uma vez que não havia a opção de filtro por índice em título ou resumo neste motor de busca.

Na plataforma ERIC tivemos alguns empecilhos, pois a mesma não possui artigos em português. Deste modo, no levantamento realizado com as palavras "sala de aula invertida" ou "aprendizagem baseada em problemas"; Resumo ou Título; Idioma (português ou inglês), até 5 anos, artigos, não obtivemos resultados. Porém, a busca com as palavras "flipped classroom" ou "problem-based learning"; no índice de Resumo; Idioma (português ou inglês), até 5 anos, artigos; foram encontrados 185 artigos. Quando substituímos o índice por Título, encontramos os mesmos 185 artigos da busca anterior, portanto estes não foram considerados. 
Tabela 3: Pesquisa primária - resultados da pesquisa no Eric. Fonte: elaboração própria.

\begin{tabular}{|c|c|c|c|c|c|}
\hline $\begin{array}{l}\text { PALAVRA- } \\
\text { CHAVE DE } \\
\text { BUSCA }\end{array}$ & $\begin{array}{l}\text { ÍNDICE } \\
\text { DE } \\
\text { BUSCA }\end{array}$ & FILTROS & $\begin{array}{c}\text { RESULT } \\
\text { A-DOS }\end{array}$ & LINK & OBSERVAÇÕES \\
\hline $\begin{array}{l}\text { "sala de aula } \\
\text { invertida" OU } \\
\text { "aprendizagem } \\
\text { baseada em } \\
\text { problemas" }\end{array}$ & resumo & $\begin{array}{l}\text { - idioma português; } \\
\text { - últimos } 5 \text { anos; } \\
\text { - artigo publicado em revista; } \\
\text { - disponível texto completo }\end{array}$ & 0 & $\begin{array}{c}\text { BUSCA } \\
13\end{array}$ & ----- \\
\hline $\begin{array}{l}\text { "sala de aula } \\
\text { invertida" OU } \\
\text { "aprendizagem } \\
\text { baseada em } \\
\text { problemas" }\end{array}$ & título & $\begin{array}{l}\text { - idioma português; } \\
\text { - últimos } 5 \text { anos; } \\
\text { - artigo publicado em revista; } \\
\text { - disponível texto completo }\end{array}$ & 0 & $\begin{array}{c}\text { BUSCA } \\
14\end{array}$ & ------- \\
\hline $\begin{array}{l}\text { "flipped classroom" } \\
\text { OR "problem-based } \\
\text { learning" }\end{array}$ & resumo & $\begin{array}{l}\text { - idioma inglês; } \\
\text { - últimos } 5 \text { anos; } \\
\text { - artigo publicado em revista; } \\
\text { - disponível texto completo } \\
\text { - revisado por pares; } \\
\text { - educação superior. }\end{array}$ & 185 & $\begin{array}{c}\text { BUSCA } \\
15\end{array}$ & \\
\hline $\begin{array}{l}\text { "flipped classroom" } \\
\text { OR "problem-based } \\
\text { learning" }\end{array}$ & título & $\begin{array}{l}\text { - idioma inglês; } \\
\text { - últimos } 5 \text { anos; } \\
\text { - artigo publicado em revista; } \\
\text { - disponível texto completo } \\
\text { - revisado por pares; } \\
\text { - educação superior. }\end{array}$ & & $\begin{array}{c}\text { BUSCA } \\
16\end{array}$ & $\begin{array}{l}\text { Os artigos são os } \\
\text { mesmos, portanto } \\
\text { estão guardados na } \\
\text { pasta } 15\end{array}$ \\
\hline
\end{tabular}

\section{TOTAL ERIC: 185}

Durante a revisão vimos em algumas plataformas um número grande de artigos. Porém, com o refino da pesquisa, pudemos buscar novos resultados, aumentando a eficiência da revisão. Do total de artigos válidos da busca primária, 564 ao todo, finalizamos a busca secundária com 10 artigos, os quais realmente discutiam acerca da aplicação dos métodos em cursos de design ou áreas afins ( 6 artigos) e em cursos técnicos ou equivalentes (4 artigos). Conforme a figura 5 abaixo, 45 artigos foram excluídos antes da busca secundária por serem repetidos.

Tabela 4: Pesquisa secundária. Fonte: elaboração própria.

\begin{tabular}{lllc}
\hline \multicolumn{2}{c}{ BUSCA PRIMÁRIA } & \multicolumn{1}{c}{ BUSCA SECUNDÁRIA } \\
\hline BUSCA 1 & 49 & $\begin{array}{l}\text { Implementação dos métodos em cursos de design ou } \\
\text { áreas afins }\end{array}$ & 1 \\
\cline { 2 - 4 } & 14 & $\begin{array}{l}\text { Implementação dos métodos em cursos técnicos ou } \\
\text { equivalentes }\end{array}$ & 1 \\
\hline BUSCA 2 & & $\begin{array}{l}\text { Implementação dos métodos em cursos de design ou } \\
\text { áreas afins }\end{array}$ & 0 \\
& & $\begin{array}{l}\text { Implementação dos métodos em cursos técnicos ou } \\
\text { equivalentes }\end{array}$ & 0 \\
\hline BUSCA 3 & 60 & $\begin{array}{l}\text { Implementação dos métodos em cursos de design ou } \\
\text { áreas afins }\end{array}$ & 0 \\
\cline { 2 - 4 } & & $\begin{array}{l}\text { Implementação dos métodos em cursos técnicos ou } \\
\text { equivalentes }\end{array}$ & 0
\end{tabular}




\begin{tabular}{|c|c|c|c|}
\hline \multirow[t]{2}{*}{ BUSCA 4} & \multirow[t]{2}{*}{29} & $\begin{array}{l}\text { Implementação dos métodos em cursos de design ou } \\
\text { áreas afins }\end{array}$ & 0 \\
\hline & & $\begin{array}{l}\text { Implementação dos métodos em cursos técnicos ou } \\
\text { equivalentes }\end{array}$ & 0 \\
\hline \multirow[t]{2}{*}{ BUSCA 6} & \multirow[t]{2}{*}{20} & $\begin{array}{l}\text { Implementação dos métodos em cursos de design ou } \\
\text { áreas afins }\end{array}$ & 0 \\
\hline & & $\begin{array}{l}\text { Implementação dos métodos em cursos técnicos ou } \\
\text { equivalentes }\end{array}$ & 1 \\
\hline \multirow[t]{2}{*}{ BUSCA 8} & \multirow[t]{2}{*}{110} & $\begin{array}{l}\text { Implementação dos métodos em cursos de design ou } \\
\text { áreas afins }\end{array}$ & 2 \\
\hline & & $\begin{array}{l}\text { Implementação dos métodos em cursos técnicos ou } \\
\text { equivalentes }\end{array}$ & 2 \\
\hline \multirow[t]{2}{*}{ BUSCA 15} & \multirow[t]{2}{*}{185} & $\begin{array}{l}\text { Implementação dos métodos em cursos de design ou } \\
\text { áreas afins }\end{array}$ & 3 \\
\hline & & $\begin{array}{l}\text { Implementação dos métodos em cursos técnicos ou } \\
\text { equivalentes }\end{array}$ & 0 \\
\hline \multirow[t]{2}{*}{ BUSCA 16} & \multirow[t]{2}{*}{52} & $\begin{array}{l}\text { Implementação dos métodos em cursos de design ou } \\
\text { áreas afins }\end{array}$ & 0 \\
\hline & & $\begin{array}{l}\text { Implementação dos métodos em cursos técnicos ou } \\
\text { equivalentes }\end{array}$ & 0 \\
\hline $\begin{array}{l}\text { TOTAL ARTIGOS } \\
\text { VÁLIDOS }\end{array}$ & 519 & TOTAL ARTIGOS VÁLIDOS & 10 \\
\hline \multirow[t]{2}{*}{$\begin{array}{l}\text { EXCLUÍDOS } \\
\text { (CRITÉRIOS DE } \\
\text { EXCLUSÃO) }\end{array}$} & 45 & $\begin{array}{l}\text { Implementação dos métodos em cursos de design } \\
\text { ou afins }\end{array}$ & 6 \\
\hline & & $\begin{array}{l}\text { Implementação dos métodos em cursos técnicos } \\
\text { ou equivalentes }\end{array}$ & 4 \\
\hline
\end{tabular}

Destes 10 artigos, há algumas considerações importantes a fazer:

1. Em todos os estudos apresentados a aplicação dos métodos resultou em números positivos, tanto no aumento da eficiência do aprendizado quanto no engajamento dos estudantes;

2. A aproximação dos alunos a problemas reais, de modo que venham adquirir conhecimento científico e tecnológico e a desenvolver o pensamento crítico e a habilidade de resolver problemas;

3. A realização de atividades em grupos pequenos de alunos e a atuação dos professores como guias ou facilitadores do processo.

Houveram resultados coletados em artigos específicos que requerem atenção. Moreira \& Lopes (2019) demonstram a importância de um acordo inicial entre as pessoas envolvidas com os novos métodos de ensino: gestores, professores e estudantes. Tal acordo funciona como apresentação do método, gera o sentimento de cooperação entre todas as partes já no começo do processo, incentivando todos a persistirem apesar dos novos desafios. Alwin \& Hussin (2018) afirmam que os estudantes que foram ensinados utilizando o método ABP aumentaram seu nível de conscientização em relação à comunidade em que vivem, já que os problemas utilizados como desafios foram problemas reais. 
Foi percebido ainda dificuldades e resistência entre os alunos na adaptação dos métodos, pois durante muito tempo os métodos pedagógicos utilizados pelas instituições de ensino ainda remontavam ao ensino tradicional. Ao romper com o ensino tradicional, percebeu-se a ansiedade dos alunos, bem como a falta de motivação diante de uma metodologia inovadora.

Tal movimento foi também verificado e discutido durante o estudo de caso realizado in loco, com turmas da disciplina Interatividade Gráfica do Curso Técnico de Computação Gráfica do IFPE - Campus Olinda (Gabriele et al., 2019). O que se verificou, com a RSL e com os estudos citados, foi um aumento da curva da eficiência acadêmica ao passo que as turmas se apropriam dos métodos ativos e se sentem mais seguros em utilizá-los.

De modo geral, a revisão sistemática da literatura demonstrou que a utilização dos métodos pedagógicos SAI e ABP possuem bons resultados quando aplicados de forma individual, mas que possuem um potencial de mudança maior quando utilizados em conjunto nas salas de aula. Os objetivos dos dois métodos são convergentes, valorizando o aprender a buscar, a selecionar e a realizar a análise crítica dos conteúdos, a se relacionar em grupo, a estabelecer relações interpessoais que fortaleçam o trabalho do grupo com atitudes cooperativas e solidárias, estando aberto a receber e a fazer críticas.

\section{Conclusões}

Aprendizagem Baseada em Problemas e Sala de Aula Invertida são métodos pedagógicos que estão sendo adotados no ensino de diversas disciplinas. Para a realização da revisão, um total de 519 artigos foram pesquisados nas bases de dados, e após a inserção do método de RSL, restaram 10 artigos. Isto nos mostra, principalmente, dois resultados importantes:

1. A aplicação dos métodos ainda é pouco estudada e observada na nossa área de atuação, e;

2. A utilização da RSL aumenta a eficiência de uma revisão de literatura, ao considerar o ganho e o aproveitamento do tempo hábil do pesquisador na análise dos artigos.

Buchanan (2014) e Moraes (2014) afirmam que o ensino do Design ainda está preso no paradigma da sociedade industrial, limitando assim o potencial reflexivo e crítico do aluno. Para Bonsiepe (2013), a mudança no ensino do Design só será viável a partir da criação de novas estruturas educacionais e adoção de experiências externas ao ambiente formal de ensino. Os métodos de ensino discutidos neste artigo adotam técnicas que apresentam aos estudantes uma nova forma de encarar os problemas propostos.

O ensino técnico e o ensino do Design tem como principal objetivo a formação de profissionais qualificados para o mercado de trabalho, logo, entende-se que a habilidade de resolver problemas seja ensinado a esses estudantes. O ensino tradicional não é mais suficiente para, no mundo complexo em que vivemos, preparar os alunos para enfrentarem os desafios diversos que envolvem encontrar soluções socialmente, economicamente, tecnologicamente, ambientalmente e culturalmente adequadas. Assim, a alternativa deve ser a 
adoção de novas metodologias de ensino, o estudo de suas aplicações, análise dos resultados obtidos e posterior divulgação para que se tornem claras as vantagens da utilização de métodos ativos, como os da SAl e da ABP.

\section{Referências}

Alwin, A. \& Hussin, R. (2018). Becoming Socially Responsible: The implementation of projectoriented problem-based learning. International Journal of Contemporary Educational Research, 5(2), 103-112.

Bishop, J. (2013). A Controlled study of the flipped classroom with numerical methods for engineers. Tese (Doutorado). UTAH State University, Logan.

Bonsiepe, G. (2013). Tendências e antitendências no design industrial. In: Moraes, D. de \& Celaschi, F. (Org.). Caderno de Estudos Avançados em Design: Design e Humanismo. 7, 61-69.

Buchanan, R. (2014). Palestra do Professor Richard Buchanan. Congresso Brasileiro de Pesquisa e Desenvolvimento em Design, 11.

Freire, P. (1987). Pedagogia do Oprimido. 17. ed. Rio de Janeiro: Paz e Terra.

Gabriele, F. P. T., Machado, A. C. S. \& Silva, E. R. V. (2019). Sala de Aula Invertida e Aprendizagem Baseada em Problemas em design: estudo de caso na disciplina "Interatividade Gráfica". Anais do Congresso Brasileiro de Design da Informação, 9, 757765. https://doi.org/10.5151/9cidi-congic-2.0123.

Galvão, T. F. \& Pereira, M. G. (2014). Revisões sistemáticas da literatura: passos para sua elaboração. Epidemiologia e Serviços de Saúde, 23, 183-184.

Jensen, C. G. (2017). Collaboration and dialogue in virtual reality. Journal of Problem Based Learning in Higher Education, 5(1).

Manzini, E. (2017). Design: quando todos fazem design. São Leopoldo: Editora Unisinos.

Moraes, D. de, Dias, M. R. A. C. (Org.) \& Sales, R. B. C. (Org.) (2014). Cadernos de Estudos Avançados em Design-Historia/History (Bilingue). Barbacena: EdUEMG.

Moreira, L. M. \& Lopes, T. I. B. (2019). Aprendizagem baseada em problemas (ABP): proposta de modelo pedagógico e avaliação da efetividade na educação profissional. Revista Brasileira da Educação Profissional e Tecnológica, 1(16), 7963.

Sampaio, R. F. \& Mancini, M. C. (2007). Estudos de revisão sistemática: um guia para síntese criteriosa da evidência científica. Brazilian Journal of Physical Therapy, 11(1), 83-89.

Souza, S. C., \& Dourado, L. (2015). Aprendizagem baseada em problemas (abp): um método de aprendizagem inovador para o ensino educativo. HOLOS, vol. 5, pp. 182-200 Instituto Federal de Educação, Ciência e Tecnologia do Rio Grande do Norte: Natal, Brasil.

\section{Sobre os autores}

Ana Carolina dos Santos Machado, Ma., IFPE, Brasil <ana.carolina@olinda.ifpe.edu.br> Elton Rubens Vieira da Silva, Me., IFPE, Brasil <elton.vieira@olinda.ifpe.edu.br>

Felipe Pessoa Tejo Gabriele, Me., IFPE, Brasil <felipe.pessoa@olinda.ifpe.edu.br> 